\title{
Da fluorescência imediata à fluorescência retardada
}

\author{
Tiago Palmeira e Mário Nuno Berberan e Santos* \\ *berberan@tecnico.ulisboa.pt
}

\begin{abstract}
From prompt to delayed fluorescence - The efficiency of emission of a photon by a molecule in a given excited state depends on the various radiative and nonradiative transition probabilities of that state. There are three molecular luminescence processes: prompt fluorescence, phosphorescence, and delayed fluorescence. Prompt fluorescence is the most common of the three. Phosphorescence can, in many cases, be observed at low temperatures (for instance $77 \mathrm{~K}$ ). Delayed fluorescence, on the other hand, is quite rare. With characteristics analogous to those of prompt fluorescence and phosphorescence, delayed fluorescence is a unique phenomenon, which can have three origins: thermal activation, triplet-triplet annihilation and charge recombination. In this paper, we discuss the characteristics and the history of delayed fluorescence by thermal activation, which is currently an important process in the area of luminescence applications.
\end{abstract}

\begin{abstract}
A eficiência de emissão de um fotão por uma molécula num dado estado excitado, depende das várias probabilidades de transição radiativas e não radiativas desse estado. Existem três processos de luminescência molecular: fluorescência imediata, fosforescência, e fluorescência retardada. A fluorescência imediata é, dos processos referidos, o que mais facilmente pode ser observado. Por sua vez, a fosforescência pode, normalmente, ser detetada a baixas temperaturas (por exemplo $77 \mathrm{~K}$ ). A fluorescência retardada é o processo menos vulgar. Com características da fluorescência imediata e da fosforescência, a fluorescência retardada é um fenómeno singular, que pode ter três origens: ativação térmica, aniquilação tripleto-tripleto e recombinação de cargas. Neste artigo, discutem-se as características e a história da fluorescência retardada por ativação térmica que é, atualmente, um processo importante nas aplicações da luminescência.
\end{abstract}

\section{Introdução}

Designa-se por luminescência a emissão espontânea de luz por uma substância que não se encontre a uma temperatura elevada (nesse caso seria radiação térmica), sendo vulgarmente designada por "luz fria" [1-3]. O termo luminescência foi introduzido em 1888 pelo físico Eilhard Wiedemann (Figura 1) após verificar que a densidade de energia da emissão de luz de uma certa substância, por unidade de comprimento de onda, era superior à que resulta da radiação térmica, dada pela lei de Kirchhoff, para a mesma temperatura [3-5]. Cada fenómeno de luminescência pode ser classificado em várias categorias de acordo com o modo de excitação utilizado (fotoluminescência, eletroluminescência, quimioluminescência, etc.). Os dois processos radiativos elementares mais importantes são a fluorescência imediata e a fosforescência. Um terceiro processo emissivo, menos comum, que apresenta características dos dois fenómenos referidos e pelo qual, recentemente, tem sido demonstrado um grande interesse, é a fluorescência retardada [2,6]. Há vários tipos de fluorescência retardada, de acordo o processo que lhe dá origem [7]: na fluorescência retardada por ativação térmica (TADF, do inglês thermally activated delayed fluorescence), o estado singuleto é populado por ativação térmica do estado tripleto; na fluorescência retardada por aniquilação tripleto-tripleto (TTA, do inglês triplet-triplet annihilation), o estado singuleto é populado pela interação de duas moléculas idênticas no estado tripleto; finalmente, na fluorescência por recombinação de cargas, o estado singuleto é populado por recombinação catião-eletrão. Destes três processos de fluorescência retardada o que ocorre por ativação térmica é o mais importante pelas suas aplicações: sensores de temperatura e oxigénio e, principalmente, díodos orgânicos emissores de luz (OLEDs, do inglês organic light emitting diode).

Neste artigo apresenta-se a TADF numa perspetiva histórica e discute-se este processo, com menção dos aspetos físicos e das diferentes aplicações, dando especial atenção

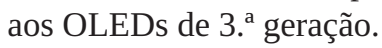

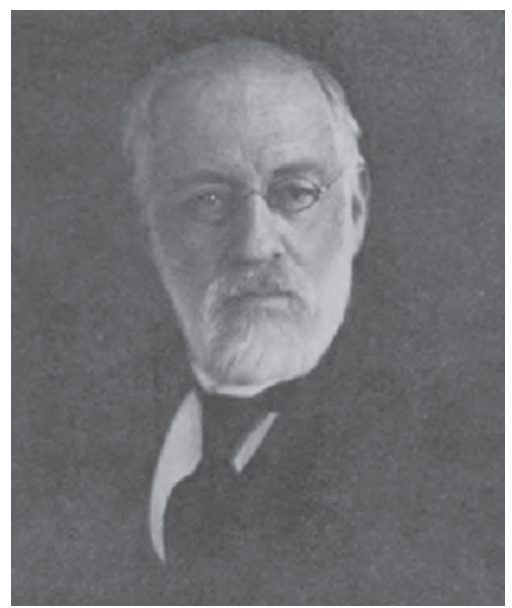

Figura 1 - Eilhard Wiedemann (1852-1928) [97].

\section{Perspetiva histórica da TADF}

Até meados do século XX, a distinção entre fluorescência e fosforescência/TADF era feita com base na duração da emissão após o fim da excitação: a fluorescência cessava imediatamente com o final da excitação, enquanto que a fosforescência/TADF durava algum tempo após o fim da 
excitação [8,9]. No que diz respeito ao processo da TADF, a sua identificação e entendimento eram mais complexos. Com os equipamentos atuais é fácil identificar a TADF enquanto tal. No entanto, o facto de a emissão ocorrer na zona espectral da fluorescência, conjugado com um tempo de vida longo, semelhante ao da fosforescência, criou, durante vários anos, alguma confusão [8-10]. Em literatura anterior a 1961 encontra-se frequentemente a TADF designada por fosforescência, fosforescência à temperatura ambiente ou fosforescência alfa. Por sua vez, a fosforescência também é designada por fosforescência de baixa temperatura, fosforescência beta ou fluorescência de longa duração [10-12]. A confusão de terminologias utilizadas tem, possivelmente, origem nos tempos de vida longos das emissões e na ausência de discriminação espectral e, por isso, era comum em estudos em que os três processos se verificavam. O primeiro investigador a observar estes três processos em simultâneo terá sido Eilhard Wiedemann, em finais do século XIX. Wiedemann contribuiu de forma muito importante para o entendimento da luminescência e, designadamente, da fosforescência. No seu artigo mais conhecido, publicado em 1888, Wiedemann descreve a transformação da fluorescência (com um tempo de vida curto) em fosforescência (com um tempo de vida longo), pela adição de gelatina, interpretando esta mudança como resultado da redução da mobilidade das moléculas [4]. Tal interpretação, apesar de não ser totalmente correta, foi aceite por mais de 40 anos. Francis Perrin (1926) e Vavilov e Levshin (1926), após os estudos dos tempos de vida de luminescência de sais de uranilo, contestaram a interpretação de Wiedemann [13,14]. Em 1892, seguindo a mesma ideia, Wiedemann publica um artigo (que poderá ser o primeiro artigo onde a observação de TADF é descrita, embora sem ser identificada enquanto tal) em que estuda a luminescência da eosina numa matriz sólida de gelatina, a altas temperaturas [15]. Utilizando um fosforoscópio construído por si, Wiedemann observa uma componente emissiva longa na zona do verde, cujo tempo de vida diminui com a temperatura, e que desaparece por completo a $200{ }^{\circ} \mathrm{C}$. De facto, para a eosina (em glicerol, por exemplo) a emissão de fluorescência ocorre no verde e a emissão de fosforescência no laranja. Mais tarde, em 1895, em colaboração com Gerhard C. Schmidt, Wiedemann publica vários artigos, onde estuda a fosforescência de diversos compostos orgânicos e inorgânicos, dissolvidos em meios viscosos e sólidos [16,17]. Desde o final do século XIX, até aos anos 20 do século XX, vários cientistas, nomeadamente James Dewar, Eugene Goldstein, Józef v. Kowalski e Erich Tiede contribuíram de forma importante para o estudo da fosforescência de corantes a baixas temperaturas [18-22].

Após a observação pioneira de Wiedemann (1892), Schmidt (1921), no decurso de estudos da luminescência de corantes orgânicos em solução e em meio sólido, observou que a cor da emissão da fluorescência dos corantes em solução era semelhante à cor da emissão de "fosforescência” (sendo a designação atual TADF) dos mesmos corantes, em meio sólido [23]. Três anos mais tarde, em 1924, Francis Perrin (Figura 2), estuda o efeito da concentração nos tempos de vida de fosforescência de corantes (eosina, eritrosina e rodamina) em soluções viscosas [24]. Um ano mais tarde, o seu pai, Jean Perrin (1925), introduz pela primeira vez o termo "fluorescência retardada” para designar uma emissão de duração semelhante à da fosforescência [25]. Em 1926, Peter Pringsheim (Figura 3) e Sergei Vavilov (Figura 4), publicaram um estudo sobre a polarização da luminescência de corantes em solução e matrizes sólidas, onde observam o aparecimento de uma emissão de "fosforescência” (designação atual: TADF) com algumas características semelhantes à da fluorescência (mesma polarização e mesma zona espectral), mas com um tempo de vida longo [26]. No entanto, é em 1929 que Francis Perrin publica o que consideramos ser um dos mais importantes estudos pioneiros da luminescência molecular: a sua dissertação de doutoramento. Nesse documento, Perrin apresenta a primeira distinção teórica entre a fluorescência e a fosforescência, ao propor um diagrama de estados eletrónicos (Figura 5), baseado na ideia de que uma molécula pode passar do estado emissivo ( $F$ ) para um estado de menor energia, designado metastável (M). Quanto ao mecanismo da fluorescência retardada, Perrin propõe que a emissão de TADF ocorre após a transição oposta, do estado metastável para o estado emissivo, por ativação térmica [27]. Pouco depois (1930), Simone Boudin publica um estudo detalhado da fosforescência da eosina em glicerol, seguindo a interpretação de Perrin, de 1924 [28]. O artigo de Boudin é referido no livro de Cecil Parker, Photoluminescence of Solutions (1968),

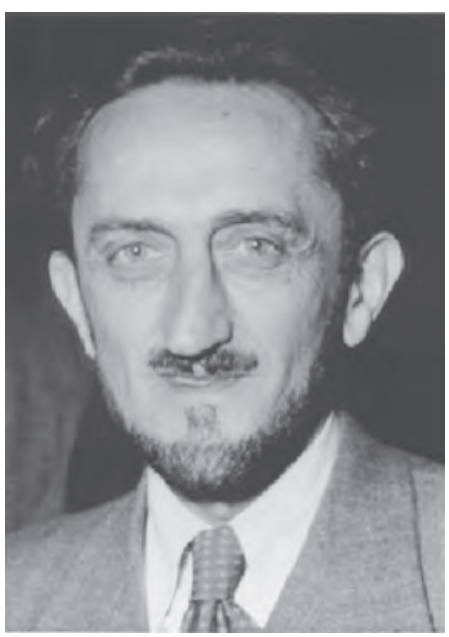

Figura 2 - Francis Perrin (1901-1992) [2].

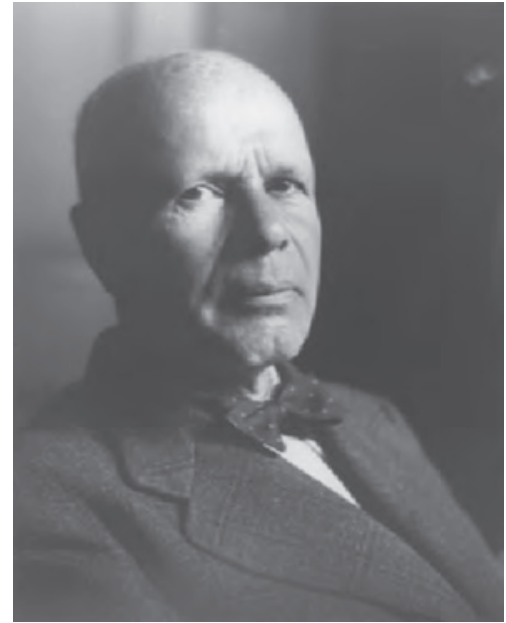

Figura 3 - Peter Pringsheim (1881-1963) [98]

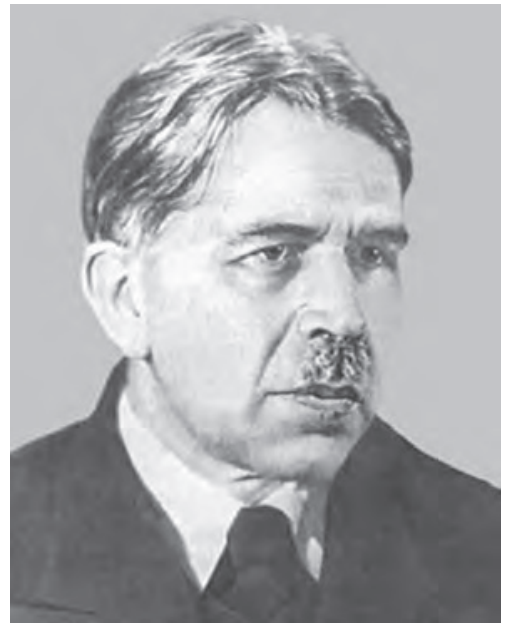

Figura 4 - Sergei Vavilov (1891-1951) [99]. 
como o primeiro artigo onde é observada a TADF [6]. Esta atribuição de prioridade, não está, como se viu, correta.

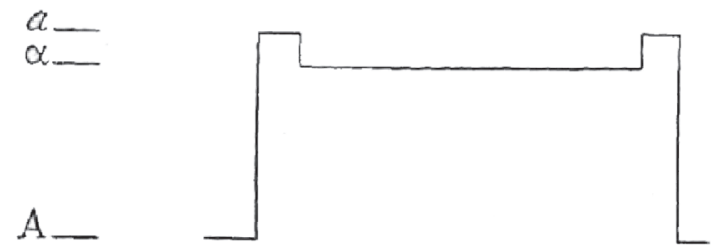

Figura 5 - Diagrama de estados e transições electrónicas, proposto por Perrin, em 1929 [27]. A - Estado Fundamental, $a$ - Estado Emissivo e $\alpha$ - Estado Metastável.

Em 1933, Alexander Jablonski (Figura 6) publica uma nota importante, na revista Nature, onde apresenta um diagrama de estados eletrónicos (ver Figura 7) que generaliza o de Perrin [29]. Tendo como base os trabalhos publicados por Hans Kautsky e colaboradores, entre 1931 e 1932, acerca do efeito do oxigénio na fluorescência de corantes [30,31], Jablonski propõe este diagrama para explicar os vários processos radiativos observados em corantes orgânicos. É de sublinhar que o esquema apresentado por Jablonski é muito semelhante ao de Perrin (1929). A grande diferença entre os dois esquemas, apresentados nas Figuras 5 e 7, é a seguinte: Jablonski considera que a emissão de fosforescência tem como origem o estado metastável. No artigo publicado em 1935, onde continua as ideias expostas no artigo de 1933, Jablonski sugere um tratamento semiquantitativo do efeito da temperatura no quociente das intensidades da fosforescência e da TADF, que permitiria obter uma energia de ativação (a partir do fator de Boltzmann) que corresponde à diferença energética entre os estados F e M (atualmente designada por $\Delta \mathrm{E}_{\mathrm{ST}}$ ), ver Figura 7 [32]. Em 1936, Levshin e Vinokurov demonstraram que os tempos de vida da TADF e da fosforescência da fluoresceína em ácido bórico eram idênticos [33]. Este resultado foi uma das primeiras evidências de que a desativação do estado metastável podia ocorrer por dois processos distintos, mas relacionados.

A confirmação experimental do tratamento exposto por Jablonski em 1935, só ocorre na década de 40, com Gilbert Lewis (Figura 8), David Lipkin e Theodore Magel (1941) [34]. Neste trabalho, Lewis, Lipkin e Magel utilizam o mesmo sistema de Levshin e Vinokurov, a fluoresceína dissolvida em ácido bórico, e medem os espectros de absorção, de luminescência (a diferentes temperaturas) e os tempos de vida de fosforescência e da TADF. A partir destes dados experimentais, constroem um diagrama semelhante ao de Jablonski (ver Figura 9) e conseguem determinar o $\Delta \mathrm{E}_{\mathrm{ST}}$ para a fluoresceína (usando as energias correspondentes aos máximos de emissão da TADF e da fosforescência - valores espectroscópicos - e através da variação da constante de velocidade da TADF com a temperatura) e confirmar que a relação de Jablonski, permitindo determinar a energia de ativação, estava essencialmente correta. Nesta altura, a natureza do estado metastável ainda era desconhecida. Em 1943, A. Terenin [35] considerou que o estado tripleto era o responsável pela fosforescência de moléculas aromáticas. Esta atribuição foi, em 1944, confirmada por Lewis e Michael Kasha [36], que apresentaram evidências de que a fosforescência tinha origem no estado tripleto, ao

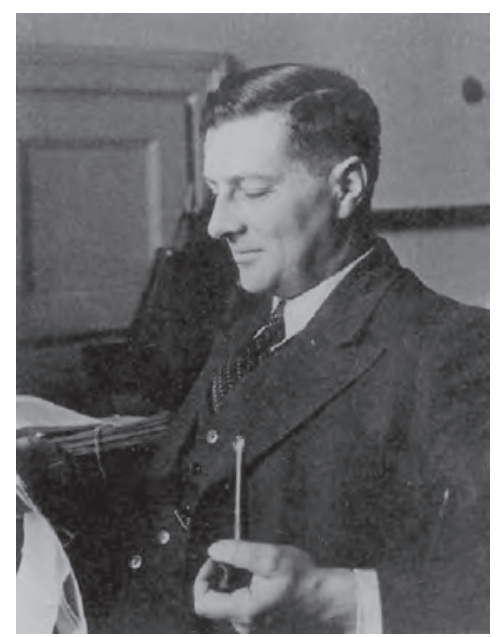

Figura 6 - Alexander Jablonski (1898-1980), adaptado de [100].

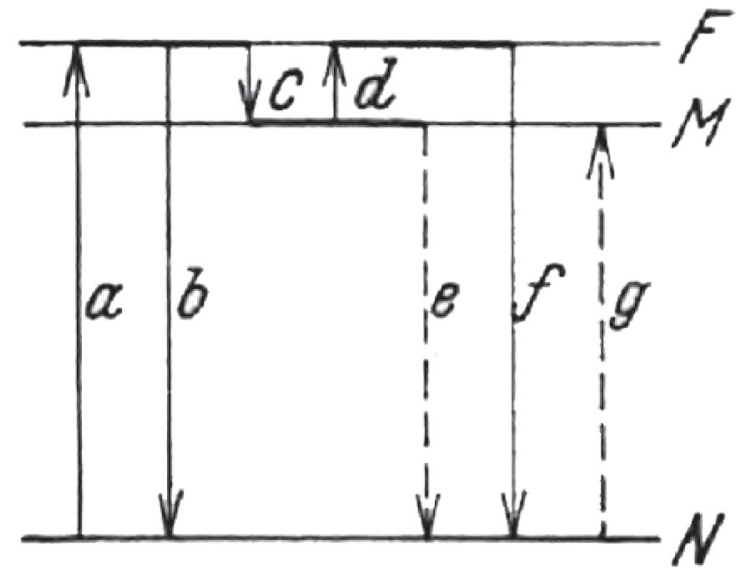

Figura 7 - Diagrama de estados e transições eletrónicas, proposto por A. Jablonski, em 1933 [29]. F - Estado Fluorescente, M - Estado Metastável, $\mathbf{N}$ - Estado Normal, $a$ - absorção, $b$ - fluorescência, $c$ - transição $\mathrm{F} \rightarrow \mathrm{M}$, $d$ - excitação térmica, $e$ - fosforescência, $f$-fosforescência (designação atual: fluorescência retardada) e $g$ - Absorção $\mathrm{M} \leftarrow \mathrm{N}$.

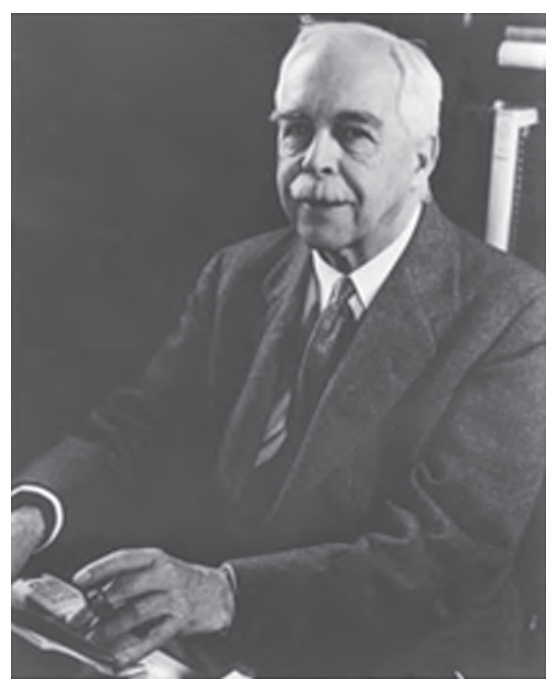

Figura 8 - Gilbert N. Lewis (1875-1946) [101].

estudar a energia das bandas da fosforescência de várias moléculas orgânicas. É também neste trabalho que Lewis e Kasha referem a investigação detalhada das propriedades do tripleto. Um ano depois, em 1945, Lewis e Calvin [37], apresentam evidência experimental para a fotossuscetibi- 
lidade magnética do tripleto, enquanto que Lewis e Kasha [38], publicam os espectros de absorção para o estado tripleto (transição $\mathrm{T}_{1} \leftarrow \mathrm{S}_{0}$ ).

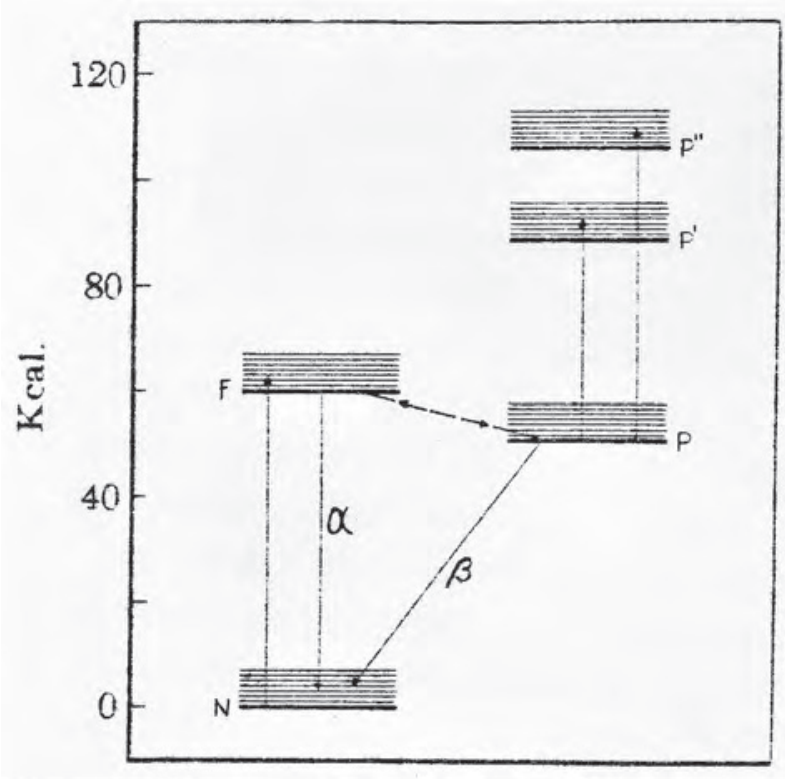

Figura 9 - Diagrama de estados e transições eletrónicas da fluoresceína em ácido bórico, proposto por Lewis, Lipkin e Magel [34]. F - Estado Fluorescente, $\mathbf{P}$ - Estado Fosforescente, $\mathbf{N}$ - Estado Normal, $\alpha$ - emissão fosforescência alfa (designação actual: TADF) e $\beta$ - emissão de fosforescência beta (designação actual: Fosforescência).

Após a identificação do estado metastável como um estado tripleto $\left(\mathrm{T}_{1}\right)$, o estudo da TADF evoluiu para a compreensão detalhada do mecanismo e para o desenvolvimento de métodos experimentais complementares dos de Lewis, Lipkin e Magel. Em particular, Rosenberg e Shombert [39] apresentaram, em 1960, um método que aperfeiçoa o de Jablonski, de 1935, baseado no quociente das intensidades da fluorescência retardada e da fosforescência, a diferentes temperaturas, para determinar o $\Delta \mathrm{E}_{\mathrm{ST}}$. Este método foi, um ano mais tarde (1961), também apresentado por Parker e Hatchard, no estudo da TADF da eosina em glicerol e etanol [40], sem citarem os autores referidos. No mesmo trabalho, Parker e Hatchard introduzem a terminologia, já referida anteriormente, para distinguir os dois tipos mais comuns de fluorescência retardada - TADF e TTA. No caso da TADF, a designação atribuída é Tipo E (de Eosina - por considerarem ter sido esta a primeira molécula em que o processo foi observado), ao passo que para a TTA, a designação escolhida é Tipo P (de Pireno) [41]. Três anos depois, em 1964, Grzywacz e Pohoski estudaram o efeito da concentração na emissão da TADF da fluoresceína em ácido bórico [42]. Em 1966, Leach e Migirdicyan, discutem de forma sistemática, as propriedades e as diferentes origens da fluorescência retardada [43]. Um ano depois, em 1967, Kropp e Dawson, apresentaram, pela primeira vez, o estudo do efeito da deuteração nos parâmetros fotofísicos da TADF, para a molécula de coroneno [44]. Em 1968, Wilkinson e Horrocks, introduziram, também pela primeira vez, a expressão «Thermally Activated Delayed Fluorescence», da qual decorre o acrónimo TADF [45]. Na década de 70, entre 1970 e 1971, Jones e Calloway apresentam o estudo da fluorescência retardada da benzofenona, dispersa em matrizes poliméricas [46,47]. No artigo de 1971, suge- rem que esta fluorescência retardada ocorre devido à ativação térmica [47]. Neste mesmo ano, Callis, Gouterman, Jones e Henderson, publicam um trabalho sobre a caracterização da TADF em porfirinas de paládio [48]. Em 1972, Brown, Singer e Parks, apresentam o estudo da TADF da benzofenona, resolvido no tempo [49]. Os dados experimentais obtidos suportam a atribuição da ativação térmica como origem para a fluorescência retardada, feita por Jones e Calloway [47]. Em 1975, Razi Naqvi e Wild estudaram a anisotropia resolvida no tempo da eosina, em polimetilmetacrilato (PMMA), propondo também a sua utilização para a quantificação da difusão rotacional de proteínas em membranas biológicas [50]. Mais tarde, em 1978, Van Der Werf, Zevenhuijzen e Jortner apresentaram um modelo teórico para lidar com a TADF de moléculas poliatómicas, em diferentes matrizes [51]. Em 1979, Greinert, Staerk, Stier e Weller, apresentaram um estudo da despolarização da TADF da eosina, para medir a difusão rotacional das proteínas inseridas em membranas [52]. Nesse mesmo ano, Garland e Moore [53], estudaram a polarização da TADF e da fosforescência, da eosina e eritrosina, quando ligada não covalentemente à albumina, em solução aquosa. Este tema é posteriormente explorado por Jovin, Bartholdi, Vaz e Austin que, em 1981, utilizam a técnica de anisotropia de fluorescência resolvida no tempo, da eosina, para caracterizar a fluidez das membranas, a partir do tempo de difusão rotacional [54]. Entre 1981 e 1984, Nishikawa e seus colaboradores apresentaram vários estudos com aplicações da TADF, observada em porfirinas e xantenos, em diferentes técnicas analíticas [55-57]. Mais tarde, em 1995, Fister, Rank e Harris publicaram um trabalho sobre a aplicação da TADF da acridina dissolvida numa matriz rígida de trealose, como termómetro ótico [58].

Em 1985, Kroto, Smalley e Curl, com a descoberta dos fulerenos [59], abriram as portas a uma nova área de investigação. Em 1996, Berberan-Santos e Garcia, demonstraram a existência de TADF no fulereno $C_{70}$ [60]. Neste trabalho, estudaram em detalhe a TADF do $\mathrm{C}_{70}$ dissolvido em parafina, utilizando dois métodos de análise: o do quociente das intensidades da TADF e da fosforescência (cuja teoria remonta a Jablonski) e o do quociente das intensidades da TADF e da fluorescência imediata. Este último método foi uma novidade relativamente aos métodos anteriores, não só porque permite o cálculo, em simultâneo, do $\Delta \mathrm{E}_{\mathrm{ST}} \mathrm{e}$ do rendimento quântico de formação do tripleto $\left(\Phi_{\mathrm{T}}\right)$ - dois parâmetros importantes na caracterização da TADF, cuja eficiência pode ser melhorada através do aumento do $\Phi_{\mathrm{T}}$ (que caracteriza a eficiência do cruzamento intersistemas) e da redução do $\Delta \mathrm{E}_{\mathrm{ST}}$ (que influencia a ativação térmica para o singuleto) - mas também pela utilização da fluorescência imediata, ao invés da fosforescência (que em certas moléculas, não é observada). Em 1997, Salazar, Fedorov e Berberan-Santos apresentaram o estudo da TADF no fulereno $\mathrm{C}_{60}$ [61]. Nesse mesmo ano, Lam e Lo, apresentaram os estudos dos tempos de vida, da TADF e da fosforescência, da eosina e da eritrosina em matriz de sílica-gel [62]. Em 1998, no seguimento do trabalho mencionado, Lam, Nambas e Lo, estudaram o efeito do oxigénio na TADF e fosforescência da eritrosina, em matriz de sílica-gel [63]. Duchowicz, Ferrer e Acuña, também em 1998, estudaram as propriedades fotofísicas e a polarização da TADF da eri- 
trosina sob condições semelhantes às condições biológicas [64]. Em 1999, Chapman, Khalil, Schibli e Gouterman, durante os estudos de moléculas luminescentes (tendo em vista a sua aplicação em tintas sensíveis à pressão do ar), observaram a TADF de uma porfirina tendo silício como átomo central [65].

Em 2000, Weisman e colaboradores apresentaram um estudo sobre as propriedades termodinâmicas e cinéticas da TADF resolvida no tempo, para derivados do $\mathrm{C}_{70}$ [66]. Mais tarde, em 2003, Weisman e colaboradores apresentaram, na continuação, um estudo sobre a TADF de derivados do $C_{60}$ [67]. Em 2006, Baleizão e Berberan-Santos apresentaram o estudo sobre a caracterização da TADF do $\mathrm{C}_{70}$, dissolvido numa matriz de poliestireno (PS) [68]. Um ano depois, em 2007, a aplicabilidade da amostra $\mathrm{C}_{70}{ }^{\prime}$ PS, como sensor de temperatura [69] e sensor de oxigénio [70], foi estudada por Nagl, Baleizão, Borisov, Schäferling, Wolfbeis e Berberan-Santos. Entre 2007 e 2010, foram publicados vários artigos sobre a TADF em fulerenos [69-75]. Em 2011, Baleizão e Berberan-Santos demonstraram, pela primeira vez, o efeito do carbono-13 $\left({ }^{13} \mathrm{C}\right)$ na eficiência da TADF do fulereno $C_{70}$. Quando enriquecido com ${ }^{13} \mathrm{C}$, o $\mathrm{C}_{70}$, apresenta um aumento da intensidade e do tempo de vida da TADF, para cerca do dobro [76]. Neste mesmo ano, Tsuboi, Penzkofer, Slyusareva e Sizykh, apresentaram os estudos sobre as propriedades fotofísicas de vários xantenos, dissolvidos em biofilmes [77]. Dois anos depois, em 2013, Kochmann, Baleizão, Berberan-Santos e Wolfbeis, estudaram a aplicabilidade do $\mathrm{C}_{70}$, enriquecido com ${ }^{13} \mathrm{C}$, como sensor de oxigénio, tendo atingidos limites de deteção extraordinários [78]. Em 2014, Palmeira, Fedorov e Berberan-Santos, apresentaram um trabalho sobre a obtenção dos parâmetros fotofísicos da TADF, com medidas resolvidas no tempo, para o fulereno $\mathrm{C}_{70}$, normal e enriquecido com ${ }^{13} \mathrm{C}$, em toda a gama de composições [79].

Até à primeira década do século XXI, a emissão de TADF estava reduzida a alguns fluoróforos, pertencentes às classes dos xantenos, porfirinas, cetonas, fulerenos e hidrocarbonetos aromáticos policíclicos (PAH, do inglês polycyclic aromatic hydrocarbons), ver Figura 10. Contudo, em 2009, Chihaya Adachi, professor da Universidade de Kyushu (Japão), propôs a aplicação da TADF em OLEDs, ditos de 3. a geração, o que desde logo motivou a síntese de novas moléculas [80-90]. Até à data (princí- pios de 2017), continuam a publicar-se, em ritmo crescente, artigos sobre novas moléculas com TADF, emitindo do azul ao vermelho (ver Figura 11) [80-90], existindo mesmo já dispositivos OLED baseados na TADF.

\section{Mecanismo da TADF}

Todos os tipos de fluorescência retardada se caracterizam por terem, simultaneamente, propriedades de fluorescência imediata e de fosforescência. A emissão dá-se sempre na zona espectral da fluorescência imediata. No entanto, o tempo de vida semelhante ao da fosforescência e a dependência linear com a intensidade de excitação são específicos da TADF (ver Figura 12) [2,5,89,90].
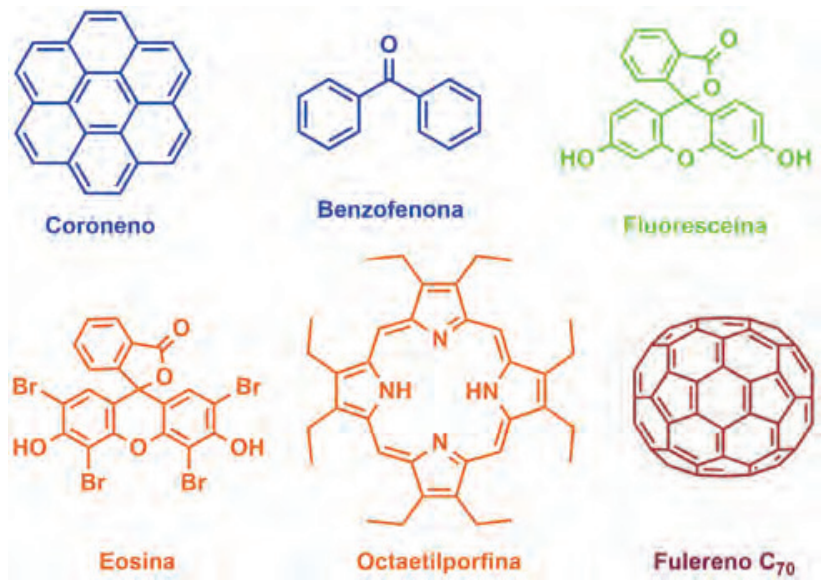

Figura 10 - Algumas moléculas com TADF identificadas até 2009.

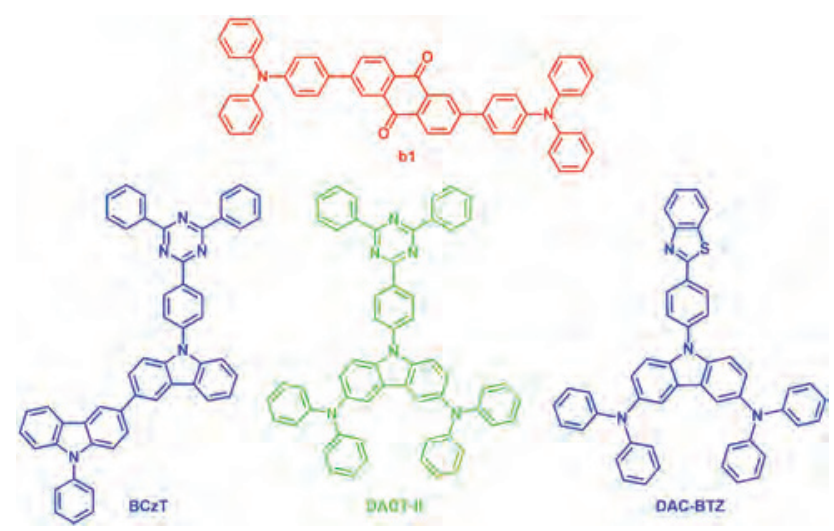

Figura 11 - Algumas moléculas com TADF, utilizadas em dispositivos OLEDs.
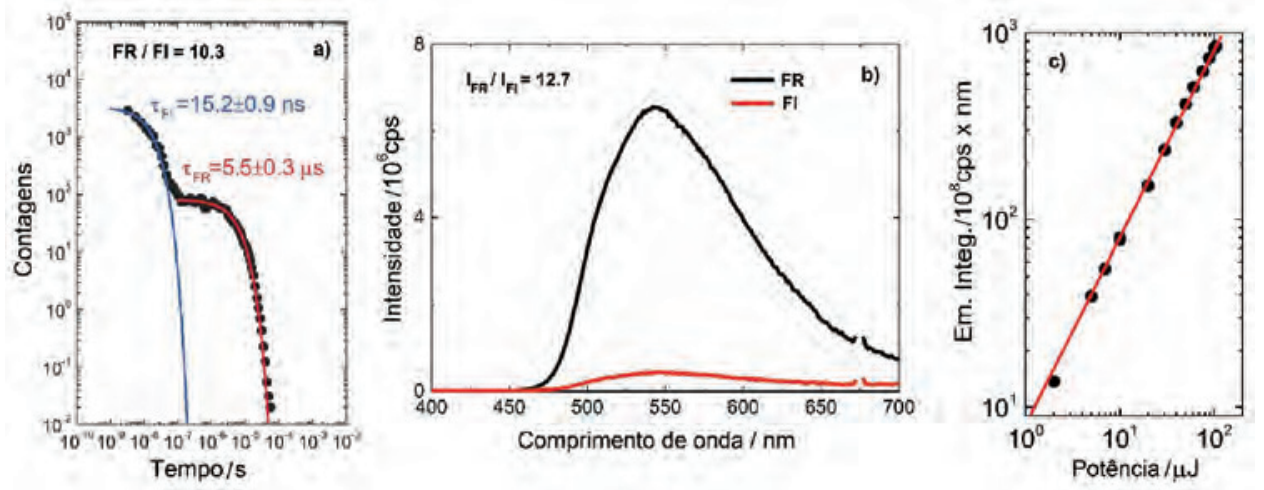

Figura 12 - a) Tempo de vida de fluorescência imediata $\left(\tau_{\mathrm{FI}}\right)$ e fluorescência retardada ( $\tau_{\mathrm{TADF}}$ ) do DPTZ-DBTO2 em metilciclo-hexano (MCH). b) Espectro de emissão da fluorescência imediata (FI), a vermelho, e da fluorescência retardada (FR), a preto, do DPTZ-DBTO2 em MCH. c) Dependência da emissão integrada da intensidade de FR com a potência de excitação para o DPTZ-DBTO2 em MCH. Adaptado de [86]. 
O mecanismo da TADF tem uma parte comum com o da fosforescência (ver Figura 13): após a promoção da molécula para um estado excitado e, assim que esta relaxa para o estado singuleto excitado de menor energia $\left(\mathrm{S}_{1}\right)$, a conversão intersistemas (CIS) para o estado tripleto (que depende do $\Phi_{T}$ ), pode ocorrer. No entanto, após a relaxação para o tripleto de menor energia $\left(\mathrm{T}_{1}\right)$, um processo de ativação térmica pode promover, de novo, a molécula para o estado $S_{1}$, por um processo que se designa por CIS inverso (que é caracterizado pelo rendimento quântico de formação do singuleto, $\left.\Phi_{S}\right)[60,83,88]$. Este processo só é efetivo sob certas condições: elevada probabilidade de CIS, pequeno $\Delta \mathrm{E}_{\mathrm{ST}}(<38 \mathrm{~kJ} / \mathrm{mol})$ e um tempo de vida de desativação do estado $\mathrm{T}_{1}$ longo (tipicamente da ordem dos microssegundos ou milissegundos) [60,91]. A somar a estas condições, a probabilidade de CIS inverso, tem também de ser elevada. Caso não o seja, a desativação para o estado fundamental pode ocorrer de forma não radiativa ou por emissão de fosforescência. Quando todas as condições anteriores são satisfeitas, a molécula, após passar de $T_{1}$ para $S_{1}$, pode decair sob a forma de fluorescência retardada ou sofrer novamente a sequência $S_{1} \rightarrow T_{1} \rightarrow S_{1}$, que depois pode dar origem à emissão. $\mathrm{O}$ número de ciclos $\mathrm{S}_{1} \rightarrow \mathrm{T}_{1} \rightarrow \mathrm{S}_{1}$ caracteriza a eficiência da TADF, e aumenta com o aumento da temperatura $[68,69,71]$.

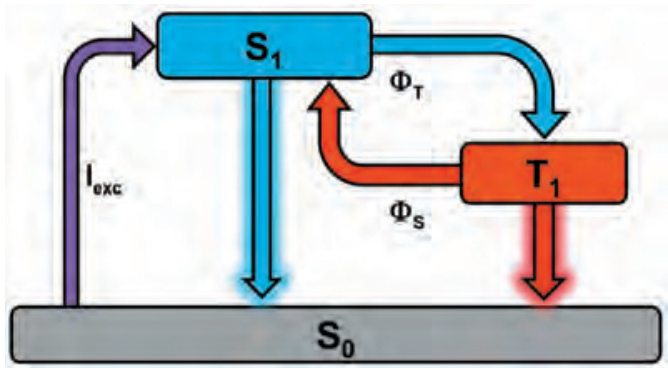

Figura 13 - Diagrama do processo de TADF, por fotoluminescência, adaptado de [91]. $\mathrm{S}_{0}$ - Estado fundamental (singuleto), $\mathrm{S}_{1}$ - Primeiro estado excitado singuleto, $\mathrm{T}_{1}$ - Primeiro estado excitado tripleto, $\mathrm{I}_{\mathrm{exc}}-\mathrm{In}$ tensidade de excitação.

Como foi já referido, até 2009 conheciam-se poucas moléculas com TADF. O elevado valor do $\Delta \mathrm{E}_{\mathrm{ST}}$, em comparação com $\mathrm{k}_{\mathrm{B}} \mathrm{T}$, é uma das principais razões para este processo ser muito pouco comum. Alguns fluoróforos, pertencentes às classes dos xantenos, porfirinas, cetonas e PAH, apresentam emissão de TADF $[41,44,46,48]$. Os fulerenos, especialmente o $\mathrm{C}_{70}$, apresentam uma eficiência de TADF extraordinária. Para o $\mathrm{C}_{70}$, a intensidade da TADF, à temperatura ambiente, é 39 vezes superior à intensidade da fluorescência imediata. Com o aumento da temperatura, graças à promoção por ativação térmica, a intensidade aumenta e, a $90{ }^{\circ} \mathrm{C}$, a TADF é 140 vezes superior à fluorescência imediata [79]. Baleizão e Berberan-Santos demonstraram pela primeira vez, em 2011, o efeito do ${ }^{13} \mathrm{C}$ na eficiência da TADF [76,95]. À temperatura ambiente a intensidade da TADF é 60 vezes superior à intensidade da fluorescência imediata. A $90{ }^{\circ} \mathrm{C}$, a TADF é 220 vezes superior à fluorescência imediata [76,95].

A partir de 2009, o número de fluoróforos com TADF aumentou muitíssimo. O desenvolvimento de OLEDs de 3. ${ }^{\text {a }}$ geração (que utilizam a emissão de TADF) levou à síntese de centenas de moléculas com TADF e emissão no visível [80-90]. Na síntese de novas moléculas, um dos principais objetivos é a minimização do $\Delta \mathrm{E}_{\mathrm{ST}}$. A elevada deslocalização dos eletrões $\pi$ e a baixa sobreposição entre a orbital molecular ocupada de maior energia (HOMO, do inglês highest occupied molecular orbital) e a orbital molecular desocupada de menor energia (LUMO, do inglês lowest unoccupied molecular orbital) são as principais causas para a diminuição do $\Delta \mathrm{E}_{\mathrm{ST}}$. Nos OLEDs de 3. a geração, as moléculas idealizadas, para obter uma TADF eficiente, apresentam uma separação espacial parcial entre a HOMO, localizada principalmente na parte dadora de eletrões, e a LUMO, localizada principalmente na parte aceitadora de eletrões. Esta separação é conseguida por aplicação de uma restrição estérica, que limita a conjugação eletrónica e conduz a um estado de transferência de carga intramolecular (ICT, do inglês intramolecular charge transfer) [80-89]. Existem moléculas com ICT que apresentam um $\Delta \mathrm{E}_{\mathrm{ST}}$ inferior a $5 \mathrm{~kJ} / \mathrm{mol}$. No entanto, como já referimos anteriormente, a emissão da TADF não depende exclusivamente do valor do $\Delta \mathrm{E}_{\mathrm{ST}}$. Outro dos parâmetros importantes, é a elevada probabilidade da CIS inversa. Este parâmetro tem um grande impacto na eficiência da emissão de TADF em OLEDs [86,88-91].

\section{Aplicações da TADF}

A presente importância da TADF resulta das suas diversas aplicações. O fabrico de dispositivos com OLEDs tem vindo a crescer e a procura de luminóforos mais eficientes, estáveis e económicos conduziu aos OLEDs de $3^{\text {a }}$ geração baseados na TADF [80-90]. No entanto, nem todas as moléculas que possuem TADF são adequadas para OLEDs. Contrariamente à fotoluminescência, em que o processo de excitação é a absorção de um fotão, na eletroluminescência, a excitação é obtida pelo movimento ordenado de cargas entre o ânodo e o cátodo [81-91]. Esta diferença nos processos de excitação faz toda a diferença na fração de moléculas que são promovidas para o estado singuleto e para o estado tripleto. Enquanto que na fotoluminescência a excitação ocorre entre os estados singuletos e a CIS se dá para o tripleto, e está dependente do valor do $\Phi_{T}$ (Figura 12), na eletroluminescência a excitação faz-se em simultâneo para singuletos e tripletos (Figura 13), sendo a probabilidade de excitação para o singuleto de $1 / 4$, e para 0 tripleto de $3 / 4$ [81-91,94]. Estas diferentes probabilidades de excitação, levam a que moléculas para aplicações fotoluminescentes necessitem de ter um elevado $\Phi_{T}$, enquanto que nas moléculas para aplicações eletroluminescentes, seja mais favorável um baixo $\Phi_{T}[91]$.

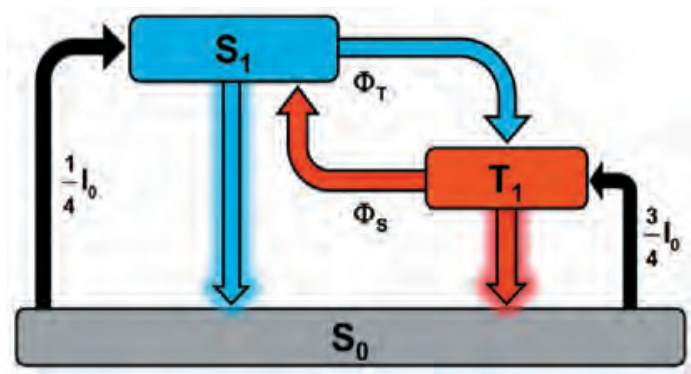

Figura 14 - Diagrama do processo de TADF, por electroluminescência, adaptado de [91]. 
Para além da aplicação em OLEDs, também é possível usar a TADF no estudo de difusão rotacional de macromoléculas biológicas e em sensores de temperatura e de oxigénio [50,51,56,67,70,72]. As membranas biológicas são estruturas dinâmicas, constituídas maioritariamente por dois tipos de moléculas: lípidos e proteínas. Consoante o seu estado físico, que é influenciado pela temperatura, pressão, hidratação e composição lipídica, as membranas podem ter propriedades dinâmicas distintas. Em meio aquoso, a difusão rotacional de macromoléculas biológicas ocorre na escala dos nanossegundos e, por isso, pode ser caracterizado pela técnica de anisotropia de fluorescência resolvida no tempo. Quando as macromoléculas são inseridas numa estrutura anisotrópica, de menor fluidez (membranas), a difusão rotacional pode ocorrer na escala dos microssegundos. Como a fluorescência imediata tem uma escala de tempo mais curta, este processo pode ser caracterizado pela anisotropia resolvida no tempo de TADF e de fosforescência, após borbulhar a solução com azoto ou árgon (para remover o $\mathrm{O}_{2}$ ) [49-51,96].

$\mathrm{O}$ desenvolvimento de sensores de temperatura e oxigénio, com base nas propriedades da TADF, foi inicialmente explorado com a acridina (apenas como sensor de temperatura), e com o fulereno $\mathrm{C}_{70}$ (como sensor dual de temperatura e oxigénio). Graças ao seu longo tempo de vida do estado tripleto (na ordem dos milissegundos) e larga gama de temperatura (de $-60{ }^{\circ} \mathrm{C}$ até $130{ }^{\circ} \mathrm{C}$ ), o $\mathrm{C}_{70}$ foi estudado como sensor de oxigénio e temperatura, tendo apresentado sensibilidade ao $\mathrm{O}_{2}$ até ao ppmv [67,70,72,95]. Posteriormente, após ter sido descrita a influência do ${ }^{13} \mathrm{C}$ nas propriedades fotofísicas (principalmente na TADF e na fosforescência) do $\mathrm{C}_{70}$, relativamente ao $\mathrm{C}_{70}$ normal, o ${ }^{13} \mathrm{C}_{70}$ foi utilizado no desenvolvimento de sensores de oxigénio ainda mais sensíveis. Com um tempo de vida, que é cerca do dobro do observado para o $\mathrm{C}_{70}$, o ${ }^{13} \mathrm{C}_{70}$ é extremamente sensível a concentrações residuais de $\mathrm{O}_{2}$ (na ordem dos ppbv) [78,95].

\section{Conclusão}

O estudo da TADF, ao longo dos anos, foi importante para a compreensão dos processos radiativos envolvendo o estado tripleto. Com propriedades simultaneamente da fluorescência e da fosforescência, este processo foi, até 2009, pouco comum e apenas observado em certos grupos de moléculas. Com o desenvolvimento dos OLEDs de 3. a geração, a TADF tem sido estudada em pormenor por vários grupos, quer da área da fotofísica, quer de materiais, tendo em vista não só uma maior compreensão do fenómeno mas também uma “engenharia molecular” eficiente.

\section{Agradecimentos}

Trabalho parcialmente financiado pelo projeto FCT FAPESP/20107/2014. T.P. é bolseiro de doutoramento da FCT (bolsa SFRH/BD/118525/2016).

\section{Referências}

[1] B. Valeur, Lumière et Luminescence; Belin: Paris, 2005.

[2] B. Valeur, M.N. Berberan-Santos, Molecular Fluorescence. Principles and Applications, $2^{\text {nd }}$ ed., Wiley-VCH: Weinheim, 2012.

[3] B. Valeur, M.N. Berberan-Santos, J. Chem. Educ. 88 (2011) 731.
[4] E. Wiedemann, Ann. Phys. 34 (1888) 446.

[5] E. Wiedemann, Ann. Phys. 38 (1889) 488.

[6] C.A. Parker, Photoluminescence of Solutions; Elsevier: Amsterdam, 1968.

[7] S.E. Braslavsky, et al.; Glossary of Terms used in Photochemistry, 3rd edition (IUPAC recommendations 2006), Pure Appl. Chem. 79 (2007) 293.

[8] E.N. Harvey, A History of Luminescence from the Earliest Times until 1900; The American Philosophical Society: Philadelphia, PA, 1957.

[9] P. Pringsheim, Fluorescence and Phosphorescence; Interscience Publishers: New York, 1949.

[10] B. Nickel, EPA Newslett. 58 (1997) 9.

[11] B. Nickel, EPA Newslett. 64 (1998) 19.

[12] S.K. Lower, M. A. El-Sayed, Chem. Rev. 66 (1966) 199.

[13] F. Perrin, Comptes Rendus 182 (1926) 219.

[14] S. Vavilov, V. Levshin, Z. Physik 35 (1926) 920.

[15] E. Wiedemann, Eder's Jahrbuch der Photog. 6 (1892) 206.

[16] E. Wiedemann, G. Schmidt, Ann.Phys. 54 (1895) 604.

[17] E. Wiedemann, G. Schmidt, Ann. Phys. 56 (1895) 18.

[18] J. Dewar, Chem. News 70 (1894) 252.

[19] J. v Kowalski, Comptes Rendus 151 (1910) 810.

[20] E. Goldstein, Physik. Z. 11 (1910) 430.

[21] E. Tiede, Chem. Ber. 53 (1920) 2214.

[22] E. Tiede, P. Wulff, Chem. Ber. 55 (1922) 588.

[23] G. Schmidt, Ann. Phys. 65 (1921) 247.

[24] F. Perrin, Comptes Rendus 178 (1924) 2252.

[25] J. Perrin, Lumière et réactions chimiques, rapport au 2e Conseil de Chimie Solvay, Bruxelles (pp 322-398 de Structure et reactivité chimique). Gauthier-Villars, Paris, 1926.

[26] P. Pringsheim, S. Vavilov, Z. Physik 37 (1926) 705.

[27] F. Perrin, Ann. Phys. 12 (1929) 169.

[28] S. Boudin, J. Chim. Phys. 27 (1930) 285.

[29] A. Jablonski, Nature 131 (1933) 839.

[30] H. Kautsky, A. Hirsch, Ber. Deutsch. Chem. Ges. 64 (1931) 2677.

[31] H. Kautsky, A. Hirsch, Ber. Deutsch. Chem. Ges. 65 (1932) 403.

[32] A. Jablonski, Z. Physik 94 (1935) 38.

[33] W. Levshin, L. Vinokurov, Physik. Z. Sowjet U. 10 (1936) 10.

[34] G.N. Lewis, D. Lipkin, T. Magel, J. Am. Chem. Soc. 63 (1941) 3005.

[35] A. Terenin, Acta Phys. Chem. URSS 18 (1943) 210.

[36] G.N. Lewis, M. Kasha, J. Am. Chem. Soc. 66 (1944) 2100.

[37] G.N. Lewis, M. Calvin, J. Am. Chem. Soc. 67 (1945) 1232.

[38] G.N. Lewis, M. Kasha, J. Am. Chem. Soc. 67 (1945) 994.

[39] J.L. Rosenberg, D. Shombert, J. Phys. Chem. 82 (1960) 3252.

[40] C.A. Parker, C. Hatchard, Trans. Faraday Soc. 57 (1961) 1894.

[41] C.A. Parker, C. Hatchard, Trans. Faraday Soc. 59 (1963) 284.

[42] J. Grzywacz, R. Pohoski, Z. Naturfarsch. 19a (1964) 440.

[43] S. Leach, E. Migirdicyan, Fluorescence a longue durée de vie de composés organiques, pp 117-186, 9a série de "Actions chimiques et biologiques des radiations” Masson: Paris (1966).

[44] J.L. Kropp, W. R. Dawson, J. Phys. Chem. 71 (1967) 4499.

[45] F. Wilkinson, A. R. Horrocks, Phosphorescence and Delayed Fluorescence of Organic Substances, pp 116-153, 
em "Luminescence in Chemistry", E. J. Bowen ed., Van Nostrand: London (1968).

[46] P.F. Jones, A.R. Calloway, J. Am. Chem. Soc. 92 (1970) 4997.

[47] P.F. Jones, A.R. Calloway, Chem. Phys. Lett. 10 (1971) 438.

[48] J.B. Callis, M. Gouterman, Y. Jones, B.H. Henderson, J. Mol. Spectrosc. 39 (1971) 410.

[49] R.E. Brown, L.A. Singer, J.H. Parks, Chem. Phys. Lett. 14 (1972) 193.

[50] K.R. Naqvi, U.P. Wild, Chem. Phys. Lett. 36 (1975) 222.

[51] R. Van Der Werf, D. Zevenhuijzen, J. Jortner, Chem. Phys. 27 (1978) 319.

[52] R. Greinert, H. Staerk, A. Stier, A. Weller, J. Biochem. Biophys. Methods 1 (1979) 77.

[53] P. Garland, C.H. Moore, Biochem. J. 183 (1979) 561.

[54] T.M. Jovin, M. Bartholdi, W.L.C. Vaz, R.H. Austin, Ann. N.Y. Acad. Sci. 366 (1981) 176.

[55] Y. Onoue, K. Hiraki, Y. Nishikawa, Bull. Chem. Soc. Japan 54 (1981) 2633.

[56] Y. Onoue, K. Hiraki, Y. Nishikawa, Bunseki Kagaku 31 (1982) 169.

[57] Y. Nishikawa, Bunseki Kagaku 33 (1984) 413.

[58] J.C. Fister, D. Rank, J.M. Harris, Anal. Chem. 67 (1995) 4269.

[59] H.W. Kroto, J.R. Heath, S.C. O’Brien, R.F. Curl, R.E. Smalley, Nature 318 (1985) 162.

[60] M.N. Berberan-Santos, J.M.M. Garcia, J. Am. Chem. Soc. 118 (1996) 9391.

[61] F.A. Salazar, A. Fedorov, M.N. Berberan-Santos, Chem. Phys. Lett. 271 (1997) 361.

[62] S.K. Lam, D. Lo, Chem. Phys. Lett. 281 (1997) 35.

[63] S.K. Lam, E. Namdas, D. Lo, J. Photochem. Photobiol. A 118 (1998) 25.

[64] R. Duchowicz, M.L. Ferrer, A. U. Acuña, Photochem. Photobiol. 68 (1998) 494.

[65] L.M. Coyle, D. Chapman, G. Khalil, E. Schibli, M. Gouterman, J. Lumin. 82 (1999) 33.

[66] S.M. Bachilo, A.F. Benedetto, R.B. Weisman, J.R. Nossal, W.E. Billups, J. Phys. Chem. A 104 (2000) 11265.

[67] S.M. Anthony, S.M. Bachilo, R.B. Weisman, J. Phys. Chem. A 107 (2003) 10674

[68] C. Baleizão, M.N. Berberan-Santos, J. Fluoresc. 16 (2006) 215.

[69] C. Baleizão, S. Nagl, S.M. Borisov, M. Schäferling, O.S. Wolfbeis, M.N. Berberan-Santos, Chem. Eur. J. 13 (2007) 3643.

[70] S. Nagl, C. Baleizão, S.M. Borisov, M. Schäferling, M.N. Berberan-Santos, O.S. Wolfbeis, Angew. Chem. Int Ed. 46 (2007) 2317.

[71] C. Baleizão, M.N. Berberan-Santos, J. Chem. Phys. 126 (2007) 204510.

[72] C. Baleizão, M.N. Berberan-Santos, Ann. NY Acad. Sci. 1130 (2008) 224.

[73] C. Baleizão, S. Nagl, M. Schäferling, M.N. Berberan-Santos, O.S. Wolfbeis, Anal. Chem. 80 (2008) 6449.

[74] C. Baleizão, M.N. Berberan-Santos, ECS Trans. 14 (2008) 3.
[75] V. Augusto, C. Baleizão, M.N. Berberan-Santos, J.P. Farinha, J. Mater. Chem. 20 (2010) 1192.

[76] C. Baleizão, M.N. Berberan-Santos, ChemPhysChem 12 (2011) 1247.

[77] T. Tsuboia, A. Penzkofer, E. Slyusareva, A. Sizykh, J. Photochem. Photobiol. A 222 (2011) 336.

[78] S. Kochmann, C. Baleizão, M.N. Berberan-Santos, O.S. Wolfbeis, Anal. Chem. 85 (2013) 1300.

[79] T. Palmeira, A. Fedorov, M. N. Berberan-Santos, Meth. Appl. Fluoresc. 2 (2014) 035002.

[80] A. Endo, M. Ogasawara, A. Takahashi, D. Yokoyama, Y. Kato, C. Adachi, Adv. Mater. 21 (2009) 4802.

[81] K. Sato, K. Shizu, K. Yoshimura, A. Kawada, H. Miyazaki, and C. Adachi, Phys. Rev. Lett. 110 (2013) 247401.

[82] Y. Tao, K. Yuan, T. Chen, P. Xu, H. Li, R. Chen, C. Zheng, L. Zhang, W. Huang, Adv. Mater. 26 (2014) 7931.

[83] C. Adachi, Jap. J. Appl. Phys. 53 (2014) 060101.

[84] D. Volz, J. Photon. Energy 6 (2016) 020901.

[85] G. Valchanov, A. Ivanova, A. Tadjer, D. Chercka, M. Baumgarten, J. Phys. Chem. A, 120 (2016) 6944.

[86] F.B. Dias, J. Santos, D. R. Graves, P. Data, R.S. Nobuyasu, M.A. Fox, A.S. Batsanov, T. Palmeira, M.N. BerberanSantos, M.R. Bryce, A.P. Monkman, Adv. Sci. 12 (2016) 1600080.

[87] S. Hirata, Y. Sakai, K. Masui, H. Tanaka, S.Y. Lee, H. Nomura, N. Nakamura, M. Yasumatsu, H. Nakanotani, Q. Zhang, K. Shizu, H. Miyazaki, C. Adachi, Nature Materials 14 (2015) 330-336.

[88] Y. Tao, K. Yuan, T. Chen, P. Xu, H. Li, R. Chen, C. Zheng, L. Zhang, W. Huang, Adv. Mater. 26 (2014) 7931.

[89] J.S. Ward, R.S. Nobuyasu, A.S. Batsanov, P. Data, A.P. Monkman, F.B. Dias, M.R. Bryce, Chem. Commun. 52 (2016) 2612.

[90] X. Cai, X. Li, G. Xie, Z. He, K. Gao, K. Liu, D. Chen, Y. Cao, S.J. Su, Chem. Sci. 7 (2016) 4264.

[91] T. Palmeira, M. N. Berberan-Santos, J. Phys. Chem. C 121 (2017) 701.

[92] M. Zander, Phosphorimetry, Academic Press: New York, 1968.

[93] S.P. McGlynn, T. Azumi, M. Kinoshita, Molecular Spectroscopy of the Triplet State, Prentice Hall: Englewood Cliffs, 1969.

[94] H. Yersin, A. Rausch, R. Czerwieniec, T. Hofbeck, T. Fischer, Coord. Chem. Rev. 255 (2011) 2622.

[95] C. Baleizão, M.N. Berberan-Santos, Química 128 (2013) 15.

[96] N.J. Turro, K-C Liu, M-F Chow, P. Lee, Photochem. Photobiol. 27 (1978) 523.

[97] H.J. Seemann, Isis 14 (1930) 166.

[98] AIP EmilioSegrè Visual Archives,https://photos.aip.org

[99] E.L. Feinberg, Physicists: Epoch and Personalities, World Scientific: Singapore, 2011.

[100] Aleksander Jablonski, J. Szudy ed., Univ. N. Kopernika: Torun, 1998.

[101] http://www.lib.berkeley.edu/uchistory/archives_exhibits/ in memoriam

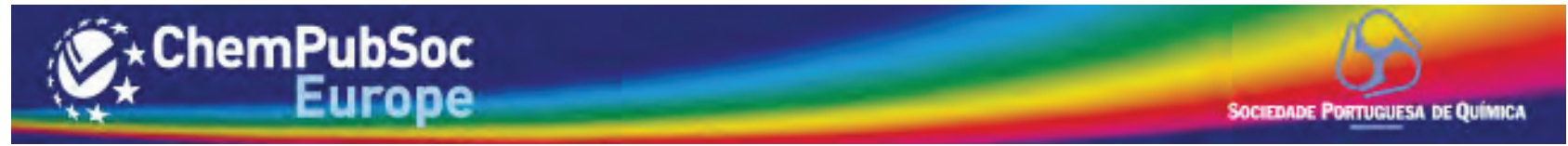

